\title{
DEVELOPMENT OF A MEMS PRECONCENTRATOR (PC) - GAS CHROMATOGRAPH (GC) FOR THE SPACECRAFT ATMOSPHERE MONITOR FOR ISS AND ORION
}

\author{
Byunghoon Bae*, Wade. G. Rellergert, Jurij Simcic, John. J. Gill, Margie L. Homer, Evan L. Neidholdt, \\ Dragan Nikolic, Richard D. Kidd, Stojan M. Madzunkov, and Murray R. Darrach \\ NASA Jet Propulsion Laboratory, California Institute of Technology, U.S.A.
}

\begin{abstract}
In this paper, we report a MEMS preconcentrator (PC) - gas chromatograph (GC) that is a crucial part of the Spacecraft Atmosphere Monitor (S.A.M.). The S.A.M. is a highly miniature gas chromatograph - mass spectrometer (GC-MS) for monitoring the atmosphere of crewed spacecraft for both trace organic compounds and the major constituents of the cabin air. The S.A.M. instrument is the next generation of GC-MS, based on JPL's Vehicle Cabin Air Monitor (VCAM), which was launched to the International Space Station (ISS) in April 2010 and successfully operated for two years $[1,2]$. The S.A.M. employs a unique MEMS PC-GC technology that replaces the macro PC-GC unit in the VCAM. We report herein the current progress of the MEMS PC-GC for the S.A.M. instrument.
\end{abstract}

\section{INTRODUCTION}

The S.A.M. instrument is JPL's atmospheric monitoring module for ISS that is based on a GC-MS system consisting of a Quadrupole Ion Trap MS (QITMS) interfaced with a MEMS PCGC unit and a small gas reservoir. The S.A.M. is intended for assessing trace volatile organic compounds and the major constituents in the atmosphere of present and future crewed spacecraft. As such, S.A.M. will continuously sample concentrations of major air constituents $\left(\mathrm{CH}_{4}, \mathrm{H}_{2} \mathrm{O}, \mathrm{N}_{2}, \mathrm{O}_{2}\right.$, and $\left.\mathrm{CO}_{2}\right)$ and report results in two-second intervals.

The S.A.M. offers further reduction in the overall instrument size to a 9.5" x 9" x 7.5" envelope, as compared to 10.8 " x 18.1 " $\mathrm{x}$ 20.4" for VCAM. This reduction in size is mainly achieved using the MEMS PC-GC unit as well as simplified electronics and other peripherals.

Trace gas analysis is based on a unique MEMS PC-GC module developed through NASA SBIR/PIDDP projects and built at JPL, which enables us to proceed with reduced power and small carrier gas consumption. A few MEMS PC-GCs have been developed before [3-5] but none of them has been devised to work with a QITMS, which requires low carrier flow (less than $0.1 \mathrm{sccm}$ ) and high vacuum-tight design $\left(10^{-8}\right.$ Torr for the JPL QITMS). Many efforts have been made to build miniaturized PC-GC systems for various applications, including homeland security and many on-site industrial applications, but no NASA mission has used MEMS PCGC technology for life support, human exploration, or planetary research. JPL has ramped up the MEMS PC-GC technology readiness level for ISS and Orion, which significantly reduces the footprint, consumables, and power.

In this paper, we present current progress of the MEMS PCGC system for the S.A.M. instrument.

\section{SYSTEM CONFIGURATION}

Figure 1(a) illustrates a conceptual schematic of the three MEMS devices - PC, microvalve (MV), and microcolumn (MC) that connects to the QITMS. Their chip footprints, shown in Fig. 1(b), are only $14 \times 14 \mathrm{~mm}^{2}$ with a 1-2 mm thickness, and they only weigh a few milligrams. To ensure that all of these devices operate properly with the QITMS, it is necessary that all the devices, their packages and fittings are vacuum-tight. Vespel housings with
Valco $^{\mathrm{TM}}$ fittings are designed and manufactured to meet this sealing requirement. Figure 1(c) shows a 3D image of a three-layer Vespel block ( $\mathrm{PC}$ top/Via/MV bottom) and indicate where $\mathrm{PC}$ and MV are inserted. By using a Via block, the PC and MV chips are separated and can be treated independently. The Via block also provides thermal isolation between the devices. Figure (d) shows an addition of the MC bottom block with the MC chip after the assembly of PC top/Via/MV bottom. This will finalize the complete assembly of PC, $\mathrm{MV}$, and $\mathrm{MC}$ with the housing blocks.

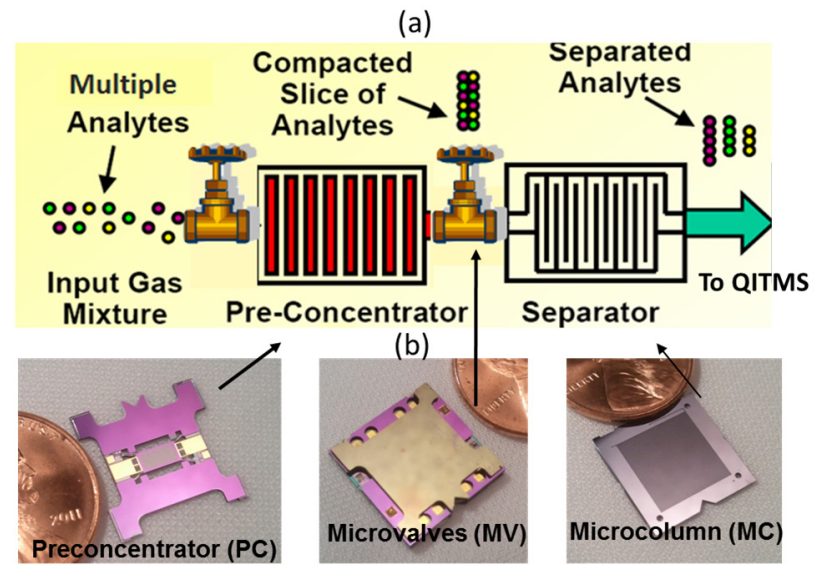

(c)
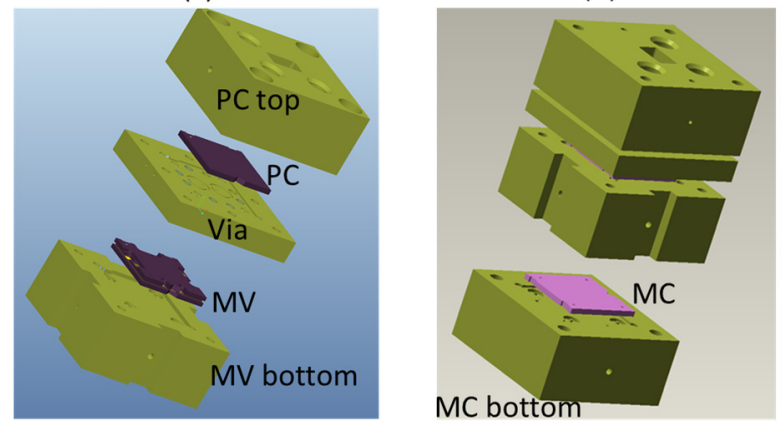

Figure 1: (a) A conceptual schematic of the MEMS PC-GC that connects to the QITMS. (b) Photographs of the PC, $M V$, and $M C$ devices compared to a U.S. penny. (c) A $3 D$ image of three-layer Vespel blocks - PC top/Via/MV bottom - as well as where PC and $M V$ are inserted. (d) An addition of the MC bottom block with the MC chip after the assembly of PC top/Via/MV bottom.

\section{PRECONCENTRATOR (PC)}

The PC uses an embedded silicon heater that employs a siliconon-insulator wafer. Due to the thermally isolated heater design as shown in Fig. 2(a), a two microliter PC chamber can be heated to $250^{\circ} \mathrm{C}$ in less than a second. Flash heating to high temperature is a key factor for a high gain PC and separation resolution in the GC downstream. Carboxen 1000 adsorbent particles are packed in the PC chamber in two layers. 
(a)

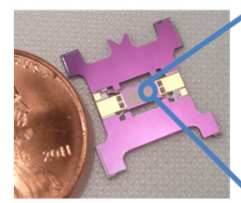

(b)

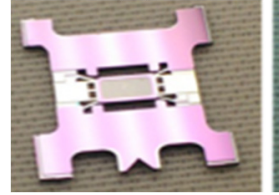

Figure 2: (a) Thermally isolated Si heater in the middle chamber where Carboxen particles a adsorbents packed (left); microposts for attaching micro/nano adsorbents (middle); Carboxen 1000 particles to be packed into the middle chamber that has no micropost (right). (b) Heater layer that is made of a silicon-oninsulator (SOI) wafer. (c) Carboxen layer where the Carboxen particles are packed (the well is on the backside), (d) Inlet/outlet layer where the gas is routed in and out.

Figure 3 shows series of acetone desorption peaks after the PC chamber adsorbs $320 \mathrm{ppm}$ of an acetone vapor sampled from a Tedlar bag for an hour. The calculated gains that count all the desorbed peaks and only the first peak are 12,000 and 4,000, respectively. Head pressure is 10 psig and a DC voltage of $85 \mathrm{~V}$ is applied for $5 \mathrm{sec}$ every 25 seconds. We find the potential of high capacity and gain for the PC since the first heating solely obtains a gain of 4,000, without including all the desorption peaks. This implies less Carboxen particles will enable PC to be completely desorbed at a single heating, which can potentially deliver the similar gain and faster heating profile.

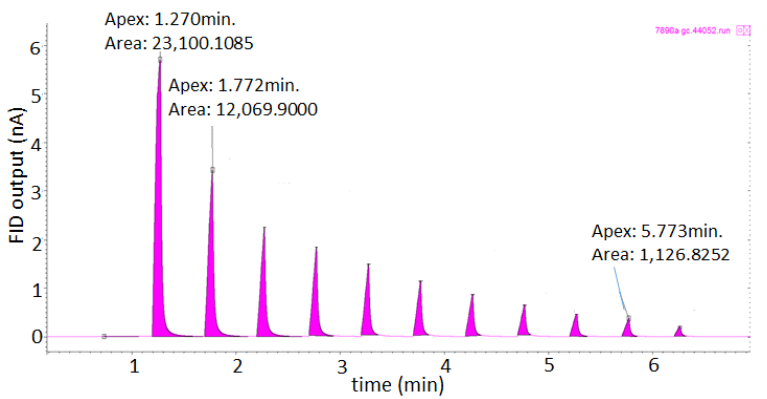

Figure 3: Periodic desorption peaks with a flame ionization detector (FID), 10 psig Helium head pressure and $85 \mathrm{~V}$ applied for 5 secs every 25 secs after 60 min of 320 ppm acetone vapor adsorption. The calculated gains that count all the desorbed peaks and only the first peak are 12,000 and 4,000, respectively.

\section{MICROVALVE (MV)}

The MV is designed to be integrated with the PC. Fig. 4(a) shows a MV schematic. The MV is composed of two highly doped Si layers that sandwich a polyimide membrane (MEM), which is electrostatically operated between valve closing/top cap (VC/TCAP) and valve opening/bottom cap (VO/BCAP) Si layers for closing and opening actions [6]. Figure 4(b) illustrates assembly of the MV layers. The VC and $\mathrm{VO}$ are bonded to TCAP and BCAP, respectively. Au hermetic diffusion bonding is used to simplify the final number of bonding layers to the MEM layer, as well as to minimize the leak rate of the MV. The MEM is bonded to VC/TCAP and $\mathrm{VO} / \mathrm{BCAP}$ using polymer adhesive bonding. The polyimide
MEM layer has four conductive layers that serve as four membrane valves: Sample (S), Vent (V), Carrier (C), and Injection (I). These independent membrane valves are integrated in the MV chip with two additional bypass (BP) valves - BP1 and BP2 - to implement complete operation of sampling and sweeping the desorbed analytes to the MC. A unique feature of this valve is the composite membrane that has an anti-stiction coating (purple color in Fig. 4(a)), a high dielectric strength, and conductive layers. Other unique features include the center pad to reduce opening voltage and charge buildup; pressure balancing (PB) mechanism to lower differential pressure across the membrane, lowering stress and allowing the valve to open against high pressure; interface treatment to prevent charge build up which is the main failure mode of most electrostatic valves [7].

(a)

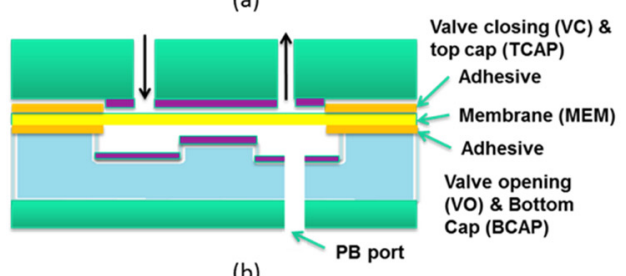

(b)

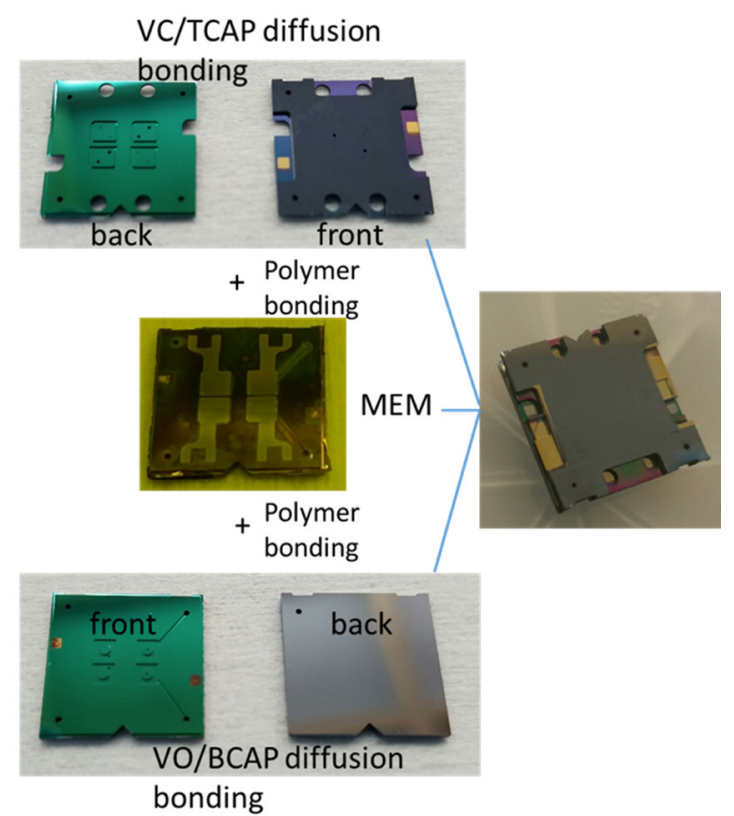

Figure 4: (a) Composition of MV layers: Two highly doped Si layers that sandwich a polyimide membrane (MEM). (b) The two highly doped layers are Au bonded stacks of valve closing/top cap (VC/TCAP) and valve opening/bottom cap (VO/BCAP) Si layers. MEM layer is electrostatically operated between VC/TCAP and VO/BCAP layers. MEM is bonded to VC/TCAP and $V O / B C A P$ using polymer adhesive bonding.

Figure 5 shows a plumbing and operational diagram of the MV that is composed of (a) sampling step, (b) desorption step, and (c) injection step. In (a), S, V, and BP2 valves are open allowing the PC to adsorb the sample by drawing the sample in using a diaphragm pump (D250SS, TCS micropumps, UK) from a Tedlar bag (or ambient). Non-adsorbed sample in the PC exits at $\mathrm{V}$. Operations of BP1/BP2 are dependent on V/I valves: BP1 is open when $\mathrm{V}$ is closed and $\mathrm{BP} 2$ valve is open when $\mathrm{I}$ is closed, and vice versa. In (b), and prior to PC heating, all the valves are closed except $\mathrm{BP} 1 / \mathrm{BP} 2$. During the heating of the PC chamber the adsorbed analyte is released but still confined to the PC. In (c), C/I/BP1 valves 
are open allowing the desorbed analyte to be swept into the column/FID by the carrier gas. PC is still heated to attain complete desorption.

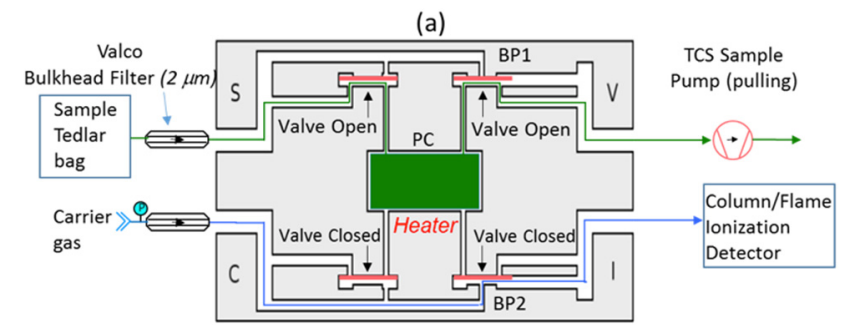

(b)

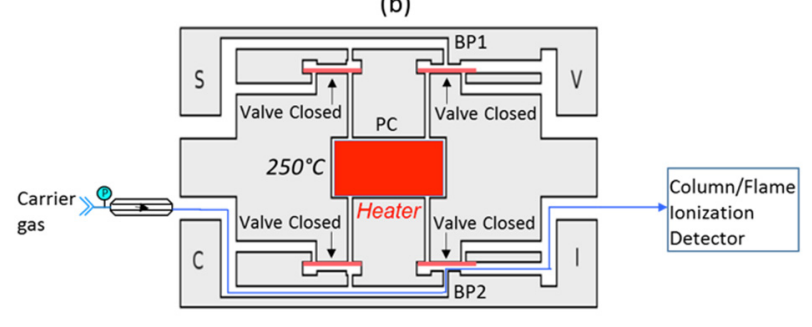

(c)

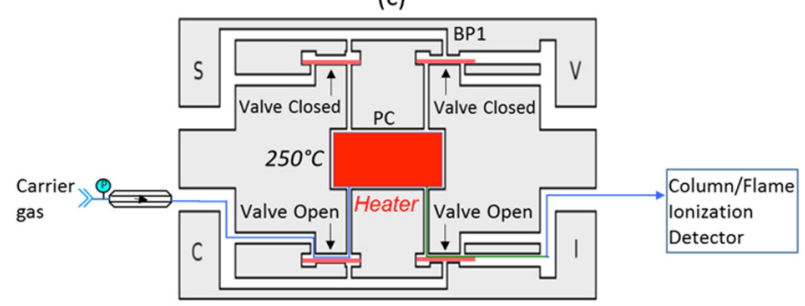

Figure 5: MV plumbing and operational diagram (a) Sampling step, (b) Desorption step, (c) Injection step.

Figure 6 shows periodic methane peaks from an FID as a MV is operated along with the steps in Fig. 5. No heating is applied. Sampling/desorption/injection steps last for $5 \mathrm{~s} / 1 \mathrm{~s} / 5 \mathrm{~s}$. DC $138 \mathrm{~V}$ is applied until 2.6 min then the power is shut off. Regular and stable injection pulse are generated, which means the MV captures the methane in the chamber and injects to the FID in a uniform and accurate way.

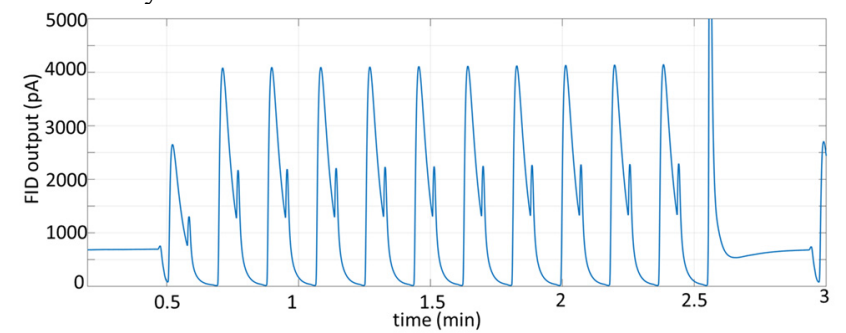

Figure 6: Periodic methane peaks from an FID as an MV is operated along with Fig. 5. Heating is not applied. Sampling/desorption/injection steps last for $5 \mathrm{~s} / 1 \mathrm{~s} / 5 \mathrm{~s} . \quad$ DC 138 $V$ is applied until 2.6 min when the power is shut off.

\section{MICROCOLUMN (MC)}

Figure 7 shows (a) a microscopic view of the serpentine channel (left) for an MC and its two-layer composition with the serpentine layer and an $\mathrm{Au}$ coated capping layer to facilitate diffusion bonding to the serpentine layer. The overall footprint of the serpentine channel is $60 \mu \mathrm{m}(\mathrm{W}) \times 100 \mu \mathrm{m}$ (D) $\times 1 \mathrm{~m}$ (L) whose hydraulic diameter is $86 \mu \mathrm{m}$. The serpentine microchannel generates better separation than that of spiral channel design in the micro level of the chip design. The waved turn geometry counteracts the

(a)

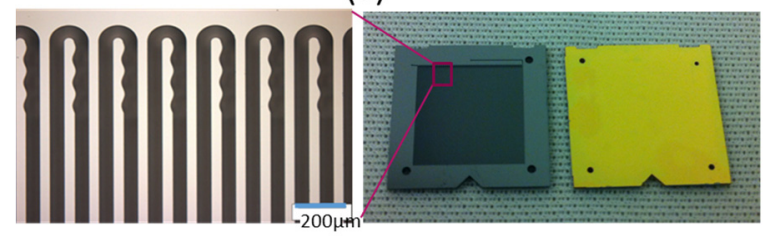

(b)

(c)

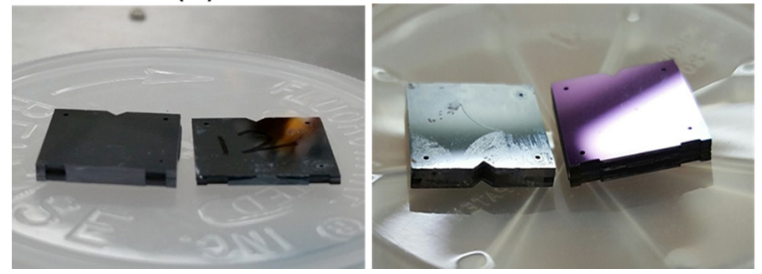

Figure 7: (a) One meter of serpentine channel whose footprint is $60 \mu \mathrm{m}(W) \times 100 \mu \mathrm{m}(\mathrm{D}) \times 1 \mathrm{~m}$ (L) (left); Au coated Si layer caps the serpentine channel layer to form an $M C$ device (right), (b) thickness comparison of $2 \mathrm{~m} v \mathrm{vs} .1 \mathrm{~m} \mathrm{MC}$, and (c) $2 \mathrm{~m}$ vs. $4 \mathrm{~m} \mathrm{MC}$.

formation of Dean Vortices, providing lower flow dispersion. To improve GC capacity and resolution, we develop $2 \mathrm{~m}$ and $4 \mathrm{~m}$ MCs by stacking two $1 \mathrm{~m}$ serpentine MCs, and having $1 \mathrm{~m}$ serpentines on both sides of two wafers, thereby eliminating the need to expand the chip platform size. Figure 7 (b) and (c) show thickness comparisons between $2 \mathrm{~m}$ and $1 \mathrm{~m}, 4 \mathrm{~m}$ and $2 \mathrm{~m}$, respectively.

Figure 8 illustrates the composition of the $2 \mathrm{~m}$ (left) and $4 \mathrm{~m}$ (right). Au diffusion bonding is used to hermetically seal all of the layers. Since the MC is the closest PC-GC component to the QITMS, hermetic sealing of the $\mathrm{MC}$ is most critical to the overall sealing of PC-GC among all the MEMS devices. Via layers are used to connect serpentine layers with the same diameter hole as the hydraulic diameter of the serpentine channel to avoid dead volume at the junction which leads to dispersion in the chromatogram.

Figure 9 illustrates a $2 \mathrm{~m} \mathrm{MC}$ chromatogram of 15 SMAC compounds using an Agilent flame ionization detector (FID). The $\mathrm{MC}$ is dynamically coated with $10 \% \mathrm{OV}-5$ (Ohio Valley, U.S.A.). A mild pressure across the $\mathrm{MC}(<10 \mathrm{psig})$ facilitates uniform coating, which improves the peak shape, tailing and dispersion. GC operating conditions are $16 \mathrm{psig}$ head pressure and $0.5 \mathrm{ml} / \mathrm{min}$ flow

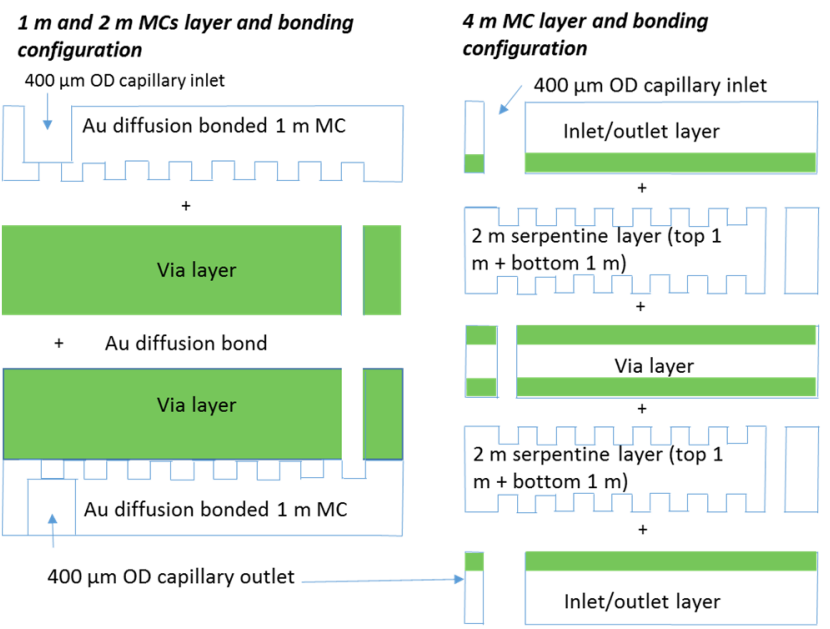

Figure 8: Configuration schematics to illustrate how $2 \mathrm{~m}$ MC can be composed of (left), and how $4 \mathrm{~m} \mathrm{MC}$ can be composed of (right). Au diffusion bonding is used to hermetically seal all the layers. 
rate. The injection volume is $0.02 \mu \mathrm{L}$ with a 500:1 split ratio.

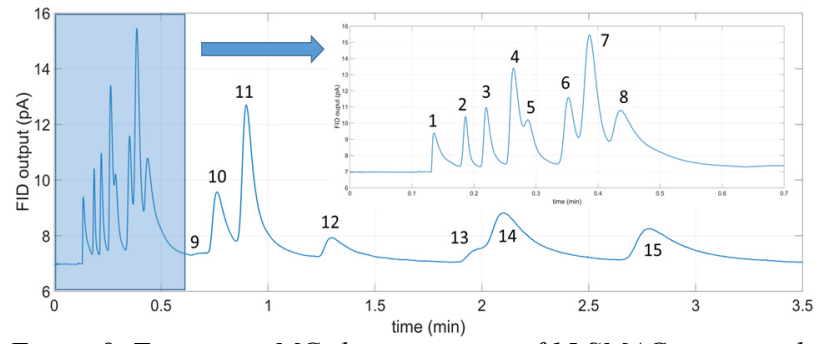

Figure 9: Two-meter MC chromatogram of 15 SMAC compounds using the Agilent FID; 15 SMAC compounds are (1) Dichloromehtane, (2) Nitromethane, (3) Chloroform, (4) 1,1,1trichloroethane, (5) 1,2-Dichloroethane (6) n-butanol, (7) Benzene, (8) Trichloroethane, (9) 2-ethoxyethanol, (10) Hexane, (11) Toluene, (12) Hexanal, (13) Diacetone alcohol, (14)Ethylbenzene, (15) O-xylene

(a)

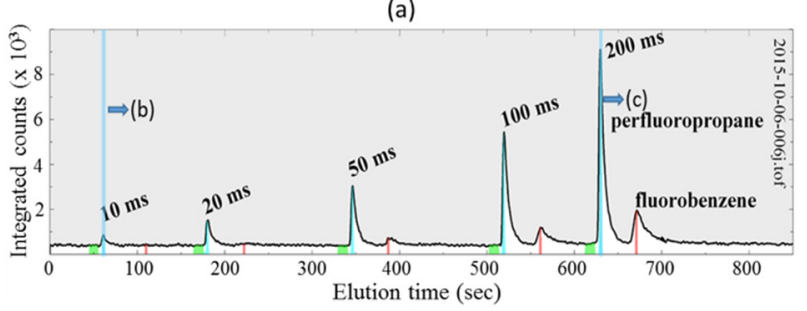

(b)

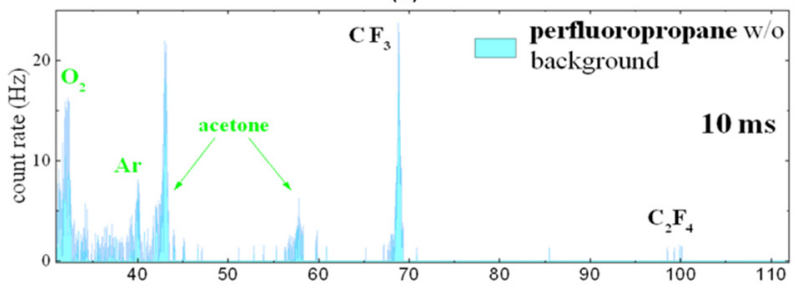

(c)

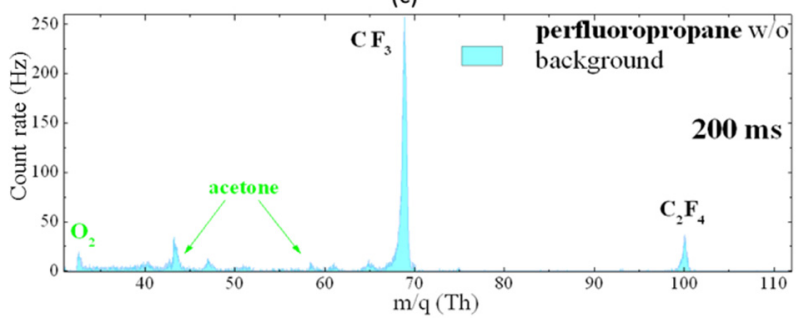

Figure 10: (a) MV operation under injections of varying duration. Elution profile exhibits five injections of perfluoropropane/fluorobenzene mixture. Injections last for 10, 20, 50, 100 and 200 milliseconds; corresponding mass spectra of perfluoropropane for shortest and longest injection times are shown in (b) and (c).

\section{INTEGRATION TESTING}

Figure 10 illustrates the results of a series of injections using the MV and sample mixture consisting of $150 \mathrm{ppm}$ of acetone, 250 ppm of perfluoropropane and $250 \mathrm{ppm}$ of fluorobenzene in helium. The sample is administered from a pressurized gas cylinder. A $1 \mathrm{~m}$ $\mathrm{MC}$ and a three-meter transfer line are connected between the MV and the QITMS. A PC is not used. Starting from the left side of plot in Fig. 10(a), subsequent injections last for 10, 20, 50, 100 and 200 $\mathrm{ms}$. The first chromatography peak corresponds to perfluoropropane, followed by a fluorobenzene peak. Figure 10(b) and (c) contain representative $\mathrm{m} / \mathrm{q}$ lines belonging to perfluoropropane and are extracted from the integral spectra by time-gating on the counts corresponding to chromatography peaks and are corrected for the underlying background spectrum. Background segments are sampled $10 \mathrm{~s}$ prior to each injection and are indicated by green color areas of elution profile found in Fig. 10(a).

The background mass spectra consists mostly of oxygen and acetone whose abundances increase twofold following each injection indicating a leak in the sample introduction line or MV packaging. Perfluoropropane and flurobenzene elutions peak are sampled in $2 \mathrm{~s}$ segments and related mass spectra for shortest and longest injection times are shown in Fig. 10(b) and 10(c), respectively. For $10 \mathrm{~ms}$ injection times, the presence of fluorobenzene is barely visible in both the elution profile and the mass spectra (not shown here), which will need the PC to boost the signal intensity. This is in sharp contrast to the perfluoropropane signal, which remains a dominant feature in both the elution profile and the $69 \mathrm{Da}$ mass spectra line.

\section{CONCLUSIONS}

We have demonstrated the individual performance of $\mathrm{PC}, \mathrm{MV}$, and $\mathrm{MC}$ as well as $\mathrm{MV} / \mathrm{MC}$ integration performance. Based on the current progress, combining all of these PC-GC devices is expected to enable a dramatic reduction in size, power consumption, and carrier gas without loss of capability for the S.A.M. instrument.

\section{ACKNOWLEDGEMENTS}

This work has been carried out at the Jet Propulsion Laboratory, California Institute of Technology, under the contract with the National Aeronautics and Space Administration.

\section{REFERENCES}

[1] A. Chutjian, et al., "Overview of the vehicle cabin atmosphere monitor, a miniature gas chromatograph/mass spectrometer for trace contamination monitoring on the ISS and CEV", SAE Technical Paper Series, 2007-01-3150, (2007).

[2] M. Darrach, et al., "Validation Test Results from the Vehicle Cabin Atmosphere Monitor", 40thInternational Conference on Environmental Systems, AIAA-2010-6094, (2010).

[3] C.-J. Lu, et al., "Functionally Integrated MEMS Micro Gas Chromatograph Subsystem," 7th Int. Conf. on Miniaturized Chemical and Biochemical Analysis Systems, Squaw Valley, CA, Oct.5-9, (2003) 411-415.

[4] S. Zampolli, et al., "Real-time monitoring of sub-ppb concentrations of aromatic volatiles with a MEMS-enabled miniaturized gas-chromatograph, "Sensors and Actuators B 141 (2009) 322-328.

[5] S. Reidy, et al., "A Micro Fabricated Comprehensive TwoDimensional Gas Chromatography System," Solid-State Sensors, Actuators, and Microsystems Workshop Hilton Head Island, SC, June 6-10 (2010) 78-81.

[6] B. Bae, et al., "A Bidirectional Electrostatic Microvalve with Microsecond Switching Performance". J. Microelectromechanical Systems, 16(6): p. 1461-1471 (2007).

[7] M. A. Shannon, B. Bae, and R. I. Masel, "Bi-Direction Rapid Action Electrostatically Actuated Microvalve," U.S. Patent 8,628,055, Jan. 14, 2014. 